\title{
Leptospirosis in pregnancy with pathological fetal cardiotocography changes
}

\author{
Suan-Li Liana Koe ${ }^{1}$, MBBs, Kim Teng $\underline{T a n}^{1}$, MMed, MRACog, Thiam Chye $\underline{T a n}^{1}$, MBBs, MMed
}

\begin{abstract}
We report the case of a 33-year-old primigravida who presented at 37 weeks of gestation with symptoms suggestive of acute fatty liver of pregnancy, but was later diagnosed with leptospirosis (i.e. Weil's disease or syndrome) on serological testing. Cardiotocography showed fetal distress, and an emergency Caesarean section was performed. A healthy neonate with no evidence of congenital leptospirosis was delivered. The patient was treated with intravenous ceftriaxone and discharged well 13 days after admission. Herein, we discuss the patient's clinical presentation and the cardiotocography changes observed in leptospiral infection, and review the current literature.
\end{abstract}

Keywords: cardiotocography, hepatitis, jaundice, leptospirosis, Weil's disease

\section{INTRODUCTION}

Leptospirosis is a zoonosis caused by the spirochaete, Leptospira interrogans. This endemic disease occurs mainly in the tropics, with some cases observed in temperate regions. In Singapore, infection is rare, with the reported incidence rate between 2002 and 2008 being $0.2-1.2$ per 100,000 persons. ${ }^{(1)}$

Leptospirosis is a biphasic disease that starts with flu-like symptoms, most (90\%) of which are mild and self-limiting. ${ }^{(2)}$ However, if left untreated, leptospirosis can progress to a more severe form (i.e. Weil's syndrome), which is characterised by jaundice, hepatorenal syndrome and haemorrhage. ${ }^{(3)}$ Mortality rates (up to $52 \%$ ) are high in the severe form of the disease. ${ }^{(2)}$

Presenting symptoms include fever, rigor, myalgia and headache (75\%-100\%); nausea, vomiting and diarrhoea (50\%); and non-productive cough $(25 \%) .{ }^{(2)}$ Other signs such as conjunctival suffusion, abdominal pain, hepatomegaly, splenomegaly, respiratory signs, skin rash and aseptic meningitis may also be present. The mean incubation period is 10 (range 2-26) days. ${ }^{(2)}$

Risk factors for infection include recreational activities (especially water-based activities and trekking), occupational exposure (e.g. work involving contact with animals, and people working as military personnel, sewer workers or laboratory workers) and household exposure (e.g. presence of domesticated animals, infestation of rodents and contaminated rainwater catchment systems). ${ }^{(3)}$ Various mammals are natural hosts for the organism, including rodents, dogs, cattle, swine, sheep and goats. Infected rodents are known to shed the organism via urine throughout life, causing environmental contamination. (2) Humans are infected after exposure to contaminated water or soil, animal urine, or diseased animal tissue. Infection is usually transmitted through direct contact, via cuts on the skin, mucous membranes or conjunctiva. Enteral and respiratory transmission is not common. ${ }^{(2)}$
Diagnosis is confirmed using cultures (from the blood, cerebrospinal fluid and urine specimens of the patient) or serology (i.e. microscopic agglutination tests, macroscopic agglutination tests, and indirect haemagglutination and enzymelinked immunosorbent assays). ${ }^{(2)}$ Laboratory abnormalities are nonspecific and include leucocytosis, elevated creatine kinase (50\% of patients) and elevations of hepatic transaminases (40\% of patients). ${ }^{(2)}$ Thrombocytopenia may be present. Urinalysis would show proteinuria, pyuria, granular casts and microscopic haematuria. (2) Hypokalaemia and hyponatraemia, with nonoliguric renal failure, may be present. (4) $^{(2)}$

Weil's syndrome is characterised by hyperbilirubinaemia, coagulopathy and renal dysfunction. Haemolytic anaemia during the second week of disease has also been reported. ${ }^{(5)}$ Rarer complications include respiratory involvement, where chest radiographs may show small nodular infiltrates progressing to consolidations. ${ }^{(2)}$

Management of the disease includes supportive measures for the liver, renal and coagulation dysfunction, and antibiotic treatment with oral doxycycline or amoxicillin. In severe disease, intravenous (IV) penicillin, doxycycline, ceftriaxone, cefotaxime or azithromycin is suggested. However, in pregnancy, doxycycline should be avoided due to permanent discolouration of teeth. The duration of treatment is usually 5-7 days. ${ }^{(2)}$

Herein, we report the case of a pregnant woman who presented at 37 weeks of gestation with jaundice, but was diagnosed with leptospirosis. To the best of our knowledge, this is the first reported case of leptospirosis in pregnancy in Singapore.

\section{CASE REPORT}

The patient was a 33-year-old primigravida with no prior medical or surgical history. She was well antenatally on regular follow-up.

${ }^{1}$ Obstetrics and Gynaecology Department, KK Women's and Children's Hospital, Singapore

Correspondence: Dr Suan-Li Liana Koe, Medical Officer, Obstetrics and Gynaecology Department, KK Women's and Children's Hospital, 100 Bukit Timah Road, Singapore 229899. lianakoe@gmail.com 


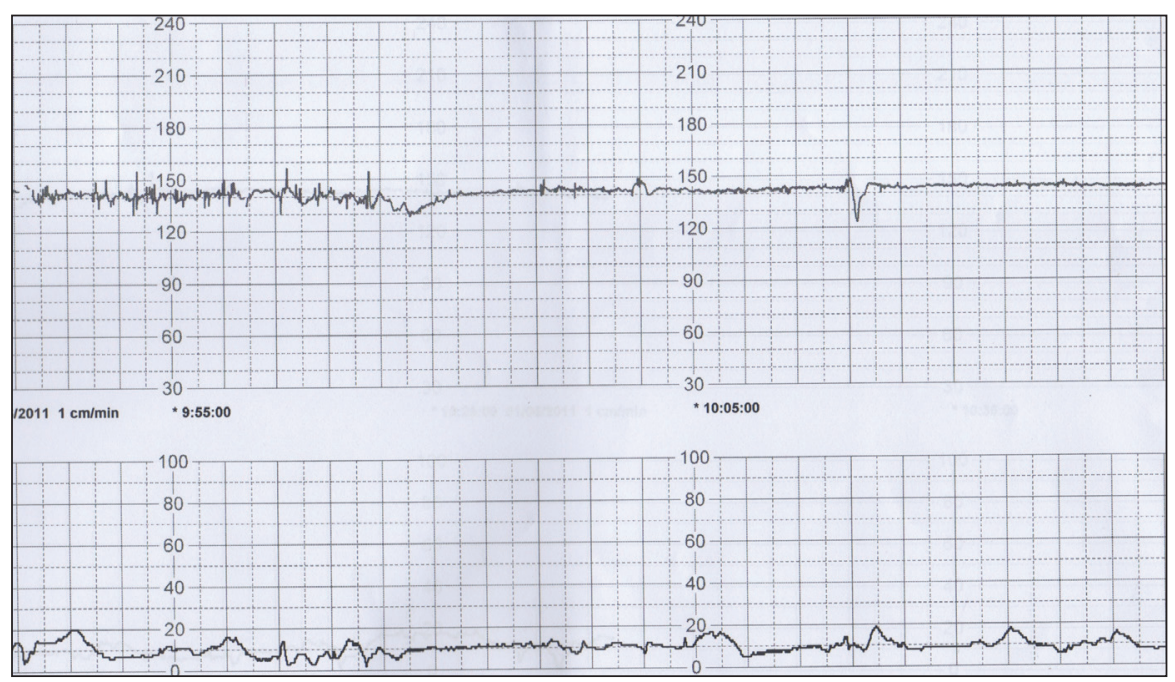

Fig. 1 Fetal cardiotocography of the patient at 37 weeks of gestation shows decreased variability, with a baseline fetal heart rate of $140 \mathrm{bpm}$.

Table I. Results of blood investigations upon admission.

\begin{tabular}{lc}
\hline Variable & Results \\
\hline Sodium (mmol/L) & 131 \\
Potassium (mmol/L) & 4.2 \\
Creatinine (umol/L) & 152 \\
Urea (mmol/L) & 3.8 \\
Haemoglobin (g/dL) & 15.3 \\
Albumin (g/L) & 24 \\
Total white blood cell count (× 10\%/L) & 15.79 \\
Platelet (× 10\%/L) & 141 \\
Neutrophil (\%) & 79 \\
Total bilirubin (umol/L) & 187 \\
Direct bilirubin (umol/L) & 103 \\
Aspartate transaminase $(\mathrm{U} / \mathrm{L})$ & 75 \\
Alanine transaminase $(\mathrm{U} / \mathrm{L})$ & 51 \\
Alkaline phosphatase $(\mathrm{U} / \mathrm{L})$ & 452 \\
Gamma-glutamyl transpeptidase (U/L) & 127 \\
Prothrombin time (s) & 23.7 \\
Partial thromboplastin time (s) & 57.6 \\
International normalised ratio & 2.20 \\
\hline
\end{tabular}

However, when seen at 35 weeks of gestation, she complained of vomiting. Clinical examination revealed she was afebrile, with a blood pressure of $128 / 78 \mathrm{mmHg}$. She was not clinically jaundiced. Bedside ultrasonography showed an estimated fetal weight of $2.5 \mathrm{~kg}$, with a normal amniotic fluid index. Fetal cardiotocography (CTG) showed a reactive trace. The patient declined admission for observation, but returned the next day with the same complaints. Clinical examination at second visit was again unremarkable and repeat CTG was reactive. The patient declined admission and was offered symptomatic treatment for vomiting.

She presented again at 37 weeks with a nine-day history of persistent vomiting, a one-day history of fever and epigastric pain, a two-day history of jaundice, and easy gum bleeding when brushing her teeth. There were no other localising symptoms of systemic infection or other symptoms of impending eclampsia such as headaches or blurring of vision. Fetal movements were good.

On clinical examination, the patient was alert, but clinically jaundiced and dehydrated. She was afebrile (temperature $36.7^{\circ} \mathrm{C}$ ), with a mildly elevated blood pressure of $146 / 95 \mathrm{mmHg}$ (mean arterial pressure $113 \mathrm{mmHg}$ ) associated with tachycardia (110 bpm). Her abdomen was soft and nontender, and Murphy's sign was negative. Reflexes were normal, with no clonus. CTG monitoring revealed poor variability, with unprovoked shallow decelerations (Fig. 1). No contractions were noted.

Based on the clinical presentation of fever, jaundice and vomiting, our initial impression was obstructive jaundice versus acute hepatitis. Initial blood tests revealed cholestatic jaundice, abnormal clotting function and impaired renal function (Table I). There was mild leucocytosis, but haemoglobin and platelet levels were normal. She was started empirically on IV ceftriaxone and metronidazole.

Continuous CTG monitoring began to show decreased variability with a fetal heart rate baseline of 140-150 bpm, associated with late decelerations (Fig. 2). The patient's cervical os was closed on vaginal examination. In view of the nonreassuring fetal status, a decision was made for an emergency Caesarean section to expedite delivery. The anaesthetic team was involved, and the patient was counselled for a Caesarean section under general anaesthesia instead of regional anaesthesia in view of the coagulopathy. An emergency lower segment Caesarean section was performed, with $1 \mathrm{~L}$ of fresh frozen plasma administered preoperatively. A healthy baby boy weighing 2,432 g was delivered, with an Apgar score of 7 at $1 \mathrm{~min}$, and 9 at 5 mins. Clear liquor was seen. Cord $\mathrm{pH}$ analysis was performed (arterial $\mathrm{pH}$ 7.236; arterial base excess 3.8; venous $\mathrm{pH}$ 7.259; venous base excess 6.0).

The intraoperative blood loss was $1 \mathrm{~L}$. Postoperatively, the patient was extubated and sent to the intensive care unit for 


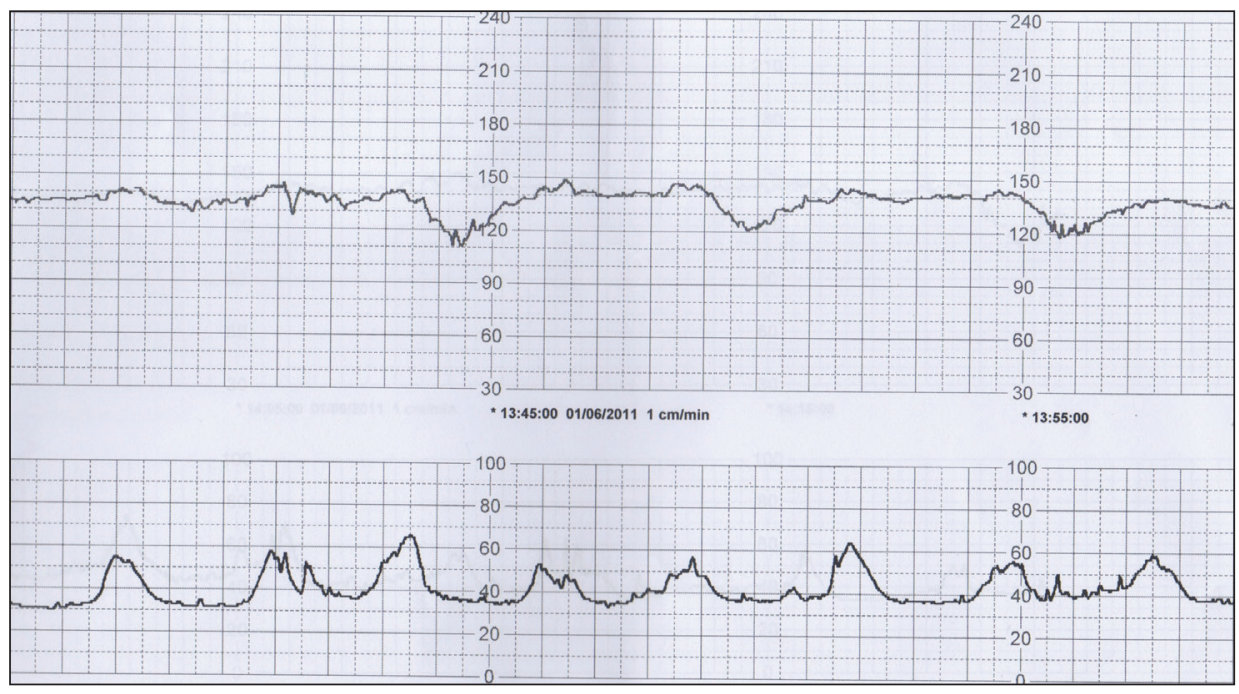

Fig. 2 Fetal cardiotocography of the patient during admission shows a pathological trace, with decreased variability and late decelerations.

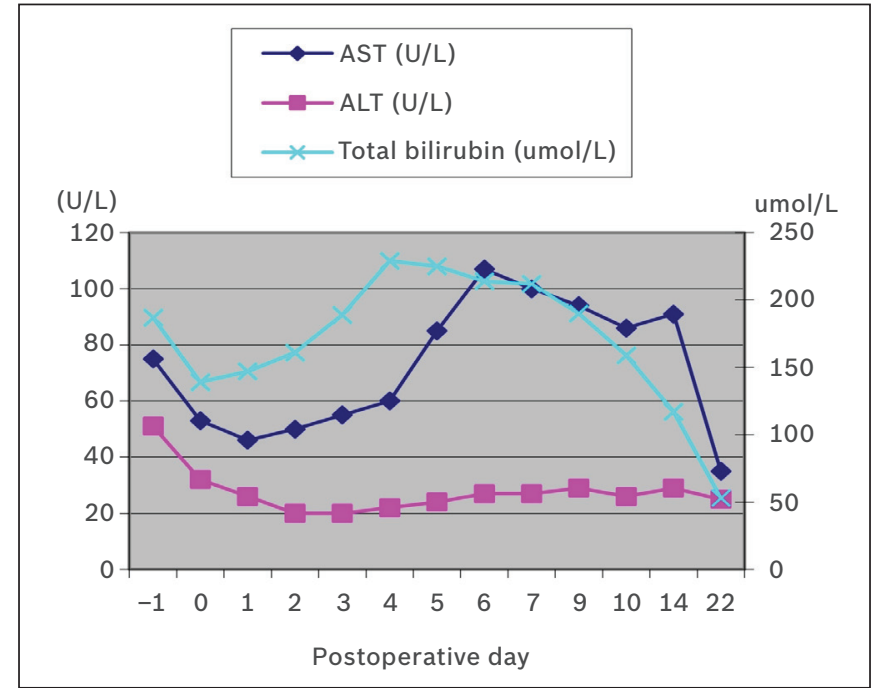

Fig. 3 Graph shows the trends in the aspartate transaminase, alanine transaminase and bilirubin levels of the patient. -1: preoperative day; 0 : operative day; ALT: alanine transaminase; AST: aspartate transaminase

further monitoring. Her liver enzymes showed mild improvement immediately after delivery, with a decrease in aspartate transaminase and alanine transaminase (Fig. 3). She was given $\mathrm{N}$-acetylcysteine for two days for liver impairment, and IV vitamin K $20 \mathrm{mg}$, two units of fresh frozen plasma and two units of platelets to correct her coagulopathy. She was continued on IV ceftriaxone and metronidazole, and remained afebrile.

On postoperative day (POD) 2, the patient developed abdominal distension secondary to postoperative ileus and ascites. Her bilirubin and aspartate transaminase levels started to increase, along with worsening coagulopathy and total white blood cell counts (Figs. 3 \& 4). Medical and gastroenterological consultations were made. Viral serology, bacterial cultures, autoimmune tests and toxicology were negative (Table II). Ultrasonography of her hepatobiliary system showed the presence of a fatty liver. Hence, acute fatty liver of pregnancy was suspected, especially in view of her rising blood pressure (systolic blood

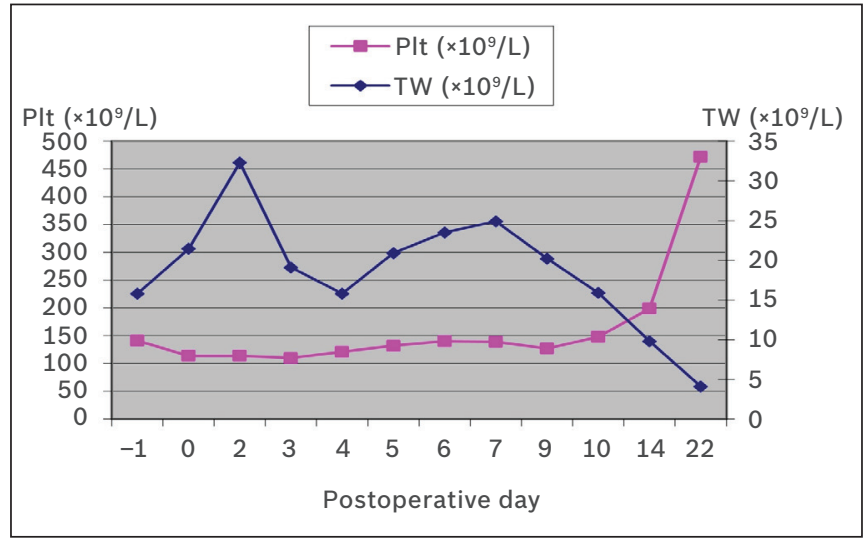

Fig. 4 Graph shows the trends in the platelet and total white blood cell counts of the patient. -1 : preoperative day; 0 : operative day; PIt: platelet; TW: total white blood cell count

pressure 160-170 $\mathrm{mmHg}$ ), proteinuria (urine protein to creatinine ratio 0.6), haemolytic anaemia (haemoglobin $10.7 \mathrm{~g} / \mathrm{L}$; lactate dehydrogenase 1,068 U/L) and mild thrombocytopenia (platelets $110 \times 10^{9} / \mathrm{L}$ ). Moderate renal impairment was attributed to dehydration. The patient was hydrated intravenously, following which creatinine levels improved. Procalcitonin and C-reactive protein levels were high (Table II), suggesting an underlying infection. Normal chest radiography ruled out chest infection. Leptospirosis serology was performed in view of the presentation of hepatorenal involvement. Leptospiral immunoglobulin $\mathrm{M}$ levels were positive on enzyme-linked immunosorbent assay serology testing, thus revealing the cause of the infection in the patient. Subsequently, treatment with IV metronidazole was stopped and IV ceftriaxone continued.

On POD 5, bilirubin levels started to fall and international normalised ratio levels improved. Improvements in aspartate transaminase level, total white blood cell count and creatinine, C-reactive protein and procalcitonin levels were seen from POD 9. Albumin and platelet levels increased from POD 10 (Figs. 3 \& 4), with normalisation of prothrombin time/partial thromboplastin time. IV ceftriaxone was stopped after 12 days 
Table II. Results of investigations during hospital stay.

\begin{tabular}{|c|c|}
\hline Variable & Results \\
\hline \multicolumn{2}{|l|}{ Hepatitis A } \\
\hline Hepatitis A total antibody & Positive \\
\hline Hepatitis A IgM & Negative \\
\hline Anti-HAV IgM & Negative \\
\hline \multicolumn{2}{|l|}{ Hepatitis B } \\
\hline Hepatitis B surface antigen & Negative \\
\hline Anti-HBc IgM & Negative \\
\hline \multicolumn{2}{|l|}{ Hepatitis C } \\
\hline Anti-HCV & Negative \\
\hline Hepatitis C total antibody* & Negative \\
\hline \multicolumn{2}{|l|}{ Ebstein-Barr virus } \\
\hline $\lg G$ & Positive \\
\hline $\operatorname{lgM}$ & Negative \\
\hline \multicolumn{2}{|l|}{ Cytomegalovirus } \\
\hline $\lg G$ & Positive \\
\hline $\operatorname{lgM}$ & Negative \\
\hline \multicolumn{2}{|l|}{ Herpes simplex virus } \\
\hline $\operatorname{lgM}$ & Negative \\
\hline \multicolumn{2}{|l|}{ Autoimmune hepatitis } \\
\hline Anti-smooth muscle & Negative \\
\hline ANA-IFA & Normal titre \\
\hline Anti-LKM (liver, kidney, muscle) & Negative \\
\hline \multicolumn{2}{|l|}{ Drug-induced } \\
\hline Toxicology screen & Negative \\
\hline Paracetamol levels & Normal \\
\hline \multicolumn{2}{|l|}{ HELLP syndrome } \\
\hline Lactate dehydrogenase (U/L) & 1,068 \\
\hline Urine protein:creatinine ratio & 0.6 \\
\hline \multicolumn{2}{|l|}{ Other investigations } \\
\hline C-reactive protein (mg/L) & 33.4 \\
\hline Procalcitonin (ug/L) & 3.34 \\
\hline Reticulocyte count (\%) & 5.7 \\
\hline Reticulocyte antibodies (109/L) & 178.9 \\
\hline 24-hr urine copper (umol/day) & 1.1 \\
\hline Total calcium (mmol/L) & 2.19 \\
\hline Magnesium (mmol/L) & 0.92 \\
\hline Inorganic phosphate (mmol/L) & 1.3 \\
\hline Caeruloplasmin (mg/L) & 224 \\
\hline Haptoglobin (mg/dL) & $<15$ \\
\hline Ferritin (ug/L) & 74 \\
\hline Amylase (U/L) & 63 \\
\hline $\lg G(g / L)$ & 16.1 \\
\hline
\end{tabular}

of treatment, and the patient was discharged well on POD 12 with oral amoxicillin/clavulanate and spironolactone for ascites treatment. Subsequent follow-up with the gastroenterologist showed normalisation of her liver enzymes on POD 22 (Fig. 3). The patient was seen in the obstetric outpatient clinic one week after discharge, with no operative complications. No significant risk factors exposing her to leptospirosis were identified.

The neonate was sent to the normal ward post delivery. On Day 3 of birth, he developed neonatal jaundice secondary to $\mathrm{ABO}$ incompatibility, requiring phototherapy. This was accompanied by raised bilirubin levels and reticulocytosis. On Day 5 of birth, his biochemical markers improved and jaundice resolved. Leptospirosis was not tested in the neonate. However, a subchorionic swab from the placenta showed no bacterial growth. Placental histology showed severe placental ischaemia with focal abruption. The infant was discharged on Day 7 of birth, and no known sequelae were identified up to two months of follow-up.

\section{DISCUSSION}

Leptospirosis in pregnancy is uncommon, with only a few published case reports. ${ }^{(6-11)}$ The presenting symptoms can vary, as previously described, and this is similarly observed even in pregnancy. Studies have reported leptospirosis in pregnant women with presentations similar to pregnancy-related liver disease. ${ }^{(6,7)}$

Pregnancy outcomes have been equally varied, including fetal loss and miscarriage (usually in within the first few months of pregnancy) ${ }_{1}^{(8,9)}$ stillbirth, ${ }^{(6)}$ congenital infection ${ }^{(8)}$ and oligohydramnios, ${ }^{(10,11)}$ as well as good outcomes with healthy neonates. ${ }^{(7,10,11)}$ Carles et al, in a case series, reported fetal death and abortion risk in more than $50 \%$ of pregnant women with leptospirosis; however, further details such as the rate of fetal transmission and sequelae in surviving neonates were unknown. ${ }^{(9)}$ Conversely, Shaked et al reported leptospirosis in a woman in the second trimester of pregnancy who delivered a healthy baby ${ }^{(8)}$ These authors also reviewed 15 reported cases of leptospirosis in pregnancy, and found that eight women had abortions, four delivered babies with signs of active infection, two delivered babies that were healthy, and the outcome of one was not stated. They concluded that spontaneous abortion was more likely if the infection occurred in the earlier months of pregnancy, but that termination of pregnancy should not be a consideration in pregnant women with leptospirosis, as congenital infection is rare. Chedraui and San Miguel reported a multipara woman at 28 weeks of gestation who presented antenatally with jaundice, fever, nausea and vomiting. ${ }^{(10)}$ Oligohydramnios was noted in the patient. She was diagnosed with leptospirosis, treated with IV penicillin $\mathrm{G}$ for five days and discharged well. Her amniotic fluid index levels returned to normal two weeks later, and she was subsequently followed up on every two weeks until the delivery of a healthy baby at term.

One study by Bolin and Koellner reported transmission via breast milk. ${ }^{(12)}$ These authors presented a case of anicteric leptospirosis in both mother and infant, and concluded that the likely route of transmission was breastfeeding. In another study by Chung et al, leptospirosis organisms were isolated from human breast milk, amniotic fluid, placenta and cord blood. ${ }^{(13)}$ As information is limited, it might be safer to withhold breastfeeding by infected mothers until the diagnosis is excluded or the mother is treated. For the same reason, neonatal jaundice in the newborns of mothers with suspected leptospirosis should be investigated for neonatal leptospiral infection. This was not done with the newborn in the present case, as the mother was diagnosed with leptospirosis only after neonatal jaundice had resolved. 
Despite the risk of neonatal leptospiral infection, none of the aforementioned reports made any mention of fetal monitoring. Only two case reports used fetal CTG at the time of diagnosis. ${ }^{(10,11)}$ In both these cases, oligohydramnios was noted, with a reassuring $\mathrm{CTG}$, and healthy neonates were delivered. In the present case, our patient had CTG abnormalities upon re-presentation in the jaundiced phase of the disease, which became nonreassuring a few hours after admission, necessitating delivery. The delivery had good neonatal outcome. CTG monitoring is the standard of care. Although there may be no CTG changes in early or mild leptospiral infection, CTG monitoring is essential in late or severe leptospiral disease, as fetal distress can be observed through CTG changes.

Even though leptospirosis is rare in Singapore, it should be suspected in patients with hepatorenal syndrome, given that the infection is endemic in the tropics. As the presentation of leptospirosis is varied, jaundice in pregnancy should be thoroughly investigated to cover all possible causes. In addition, in view of the potentially poor pregnancy outcomes of stillbirth and miscarriage associated with leptospirosis in pregnancy, fetal CTG monitoring seems appropriate, especially in late pregnancy and severe stages of the disease. Timely detection of fetal distress would aid in prompt delivery, and ensure optimal maternal and fetal outcomes.

\section{REFERENCES}

1. Ministry of Health, Singapore. Communicable Diseases Surveillance in Singapore 2010. Singapore: Ministry of Health, Singapore, 2011.

2. Everett ED, Calderwood SB, eds. Microbiology, epidemiology, clinical manifestations, and diagnosis of leptospirosis. In: UpToDate [online]. Available at: www.uptodate.com. Accessed September 6, 2011.

3. Farr RW. Leptospirosis. Clin Infect Dis 1995; 21:1-6.

4. Wu MS, Yang CW, Pan MJ, Chang CT, Chen YC. Reduced renal $\mathrm{Na}^{+}-$ $\mathrm{K}^{+}-\mathrm{Cl}^{-}$co-transporter activity and inhibited NKCC2 mRNA expression by Leptospira shermani: from bed-side to bench. Nephrol Dial Transplant 2004; 19:2472-9.

5. Zschiedrich S, Fischer KG. Severe Weil's syndrome complicated by hemolytic anemia. Int J Infect Dis 2010; 14:e181-2.

6. Baytur YB, Lacin S, Koyuncu FM, et al. Weil's syndrome in pregnancy. Eur J Obstet Gynecol Reprod Biol 2005; 119:132-3.

7. Gaspari R, Annetta MG, Cavaliere F, et al. Unusual presentation of leptospirosis in the late stage of pregnancy. Minerva Anestesiol 2007; 73:429-32.

8. Shaked Y, Shpilberg O, Samra D, Samra Y. Leptospirosis in pregnancy and its effect on the fetus: case report and review. Clin Infect Dis 1993; $17: 241-3$.

9. Carles G, Montoya E, Joly F, Peneau C. [Leptospirosis and pregnancy. Eleven cases in French Guyana]. J Gynecol Obstet Biol Reprod (Paris) 1995; 24:418-21. French.

10. Chedraui PA, San Miguel G. A case of leptospirosis and pregnancy. Arch Gynecol Obstet 2003; 269:53-4.

11. Dadhwal V, Bahadur A, Deka D. Leptospirosis as a cause of fever in pregnancy. Int J Gynaecol Obstet 2007; 99:252-3.

12. Bolin CA, Koellner P. Human-to-human transmission of Leptospira interrogans by milk. J Infect Dis 1988; 158:246-7.

13. Chung HL, Tsao WC, Mo PS, Yen C. Transplacental or congenital infection of leptospirosis. Chin Med J (Engl) 1963; 82:777-82. 